\title{
A/G-Specific Adenine DNA Glycosylase
}

National Cancer Institute

\section{Source}

National Cancer Institute. A/G-Specific Adenine DNA Glycosylase. NCI Thesaurus. Code C61059.

A/G-specific adenine DNA glycosylase protein (546 aa, $\sim 60 \mathrm{kDa}$ ) is encoded by the MUTYH gene. This nuclear enzyme plays a role in mismatch repair via the removal of mutagenic adenine/8-oxo-guanine. 